\title{
Pneumomédiastin post-traumatique isolé
}

\section{Isolated Post-Traumatic Pneumomediastinum}

\author{
M. Poncet $\cdot$ J. Schmidt $\cdot$ F. Moustafa
}

Reçu le 8 juin 2018; accepté le 8 mai 2019

(C) SFMU et Lavoisier SAS 2019

Un homme de 19 ans s'est présenté aux urgences pour une douleur sternale, 24 heures après un impact direct lors d'un match de basket. L'hémodynamique était stable, le patient était eupnéique avec une saturation en oxygène à $100 \%$ en air ambiant. Une radiographie thoracique et du sternum ont été réalisées. Le praticien en charge du patient ne notait pas de lésion visualisable et laissait le patient regagner son domicile. La relecture du cliché le lendemain a mis en évidence un pneumomédiastin associé à un emphysème sous-cutané du creux sus-claviculaire (Fig. 1A). Le patient a donc été recontacté afin de réaliser un scanner thoracique sans injection. Cette imagerie a confirmé le diagnostic de pneumomédiastin de moyenne abondance avec extension cervicale et sous-cutanée sans étiologie retrouvée (pas de lésion osseuse traumatique, pas de brèche trachéale ni de perforation œsophagienne visible sur cet examen) (Fig. 1BC). L'indication d'endoscopie bronchique ou de scanner avec opacification digestive, afin de rechercher une brèche trachéale ou une perforation œsophagienne, n'a pas été retenue chez ce patient. Il a été autorisé à rentrer chez lui avec une prescription d'antalgiques simples et de repos. L'évolution a été favorable.

Le pneumomédiastin est défini par la présence d'air à l'intérieur du médiastin dont l'origine peut être spontanée ou secondaire. Les deux étiologies graves à rechercher

\footnotetext{
M. Poncet $(\bowtie) \cdot J$. Schmidt $\cdot$ F. Moustafa

Université Clermont-Auvergne, UFR de médecine,

28, place Henri-Dunant,

F-63001 Clermont-Ferrand cedex 01, France

e-mail : marion.poncet@laposte.net

M. Poncet

Service des urgences, centre hospitalier du Puy-en-Velay,

12, boulevard Docteur-Chantemesse,

F-43000 Le Puy-en-Velay, France

J. Schmidt $\cdot$ F. Moustafa

Service des urgences,

centre hospitalier universitaire de Clermont-Ferrand,

58, rue Montalambert, F-63000 Clermont-Ferrand, France
}

sont une rupture œsophagienne (spontanée : syndrome de Boerhaave) et une lésion de l'arbre trachéobronchique (post-traumatique et à forte cinétique le plus souvent). Le pneumomédiastin est principalement décrit dans des contextes post-traumatiques (traumatisme thoracique fermé ou pénétrant, traumatisme facial). Il peut aussi être secondaire à une chirurgie maxillofaciale, dentaire (par diffusion de l'air sous pression de la cavité buccale vers le médiastin), digestive ou à d'autres facteurs favorisants (crise d'asthme, effort physique, inhalation de drogues, répétition de vomissements).

Le mécanisme physiopathologique à l'origine du pneumomédiastin traumatique est appelé l'effet Macklin [1] : il s'agit d'une rupture alvéolaire par hyperpression intraalvéolaire, entraînant une dissection de l'interstitium pulmonaire puis la diffusion de l'air le long des gaines conjonctives péribronchovasculaires jusque dans le médiastin.

La symptomatologie clinique retrouve souvent une douleur thoracique, une dyspnée et un emphysème sous-cutané cervical ou thoracique. Le signe de Hamman en est pathognomonique (présent une fois sur deux) et doit être recherché : il s'agit de bruits de crépitations à l'auscultation de l'aire précordiale synchrones aux bruits du cœur. D'autres symptômes tels que la toux, une dysphagie, une dysphonie peuvent être présents. Fréquemment, un pneumothorax peut être associé.

L'examen réalisé en première intention est la radiographie thoracique. Plusieurs signes radiologiques [2] sont à rechercher. On recherche la bande d'hyperclarté des contours du médiastin parallèles aux contours du cœur et de l'aorte thoracique descendante. La présence d'air dans les parties molles du cou ou du thorax en faveur d'un emphysème souscutané peut aussi nous orienter sur le diagnostic. Un autre signe radiologique : le signe de la coupole diaphragmatique continue, appelé signe de Levine, est observé lorsque l'air s'interpose entre le cœur et le diaphragme, et permet de visualiser la portion centrale de la coupole gauche. Enfin, le signe du thymus volant ou du spinnaker est également décrit : il s'agit de la surélévation thymique en aile d'ange par l'air médiastinal. 

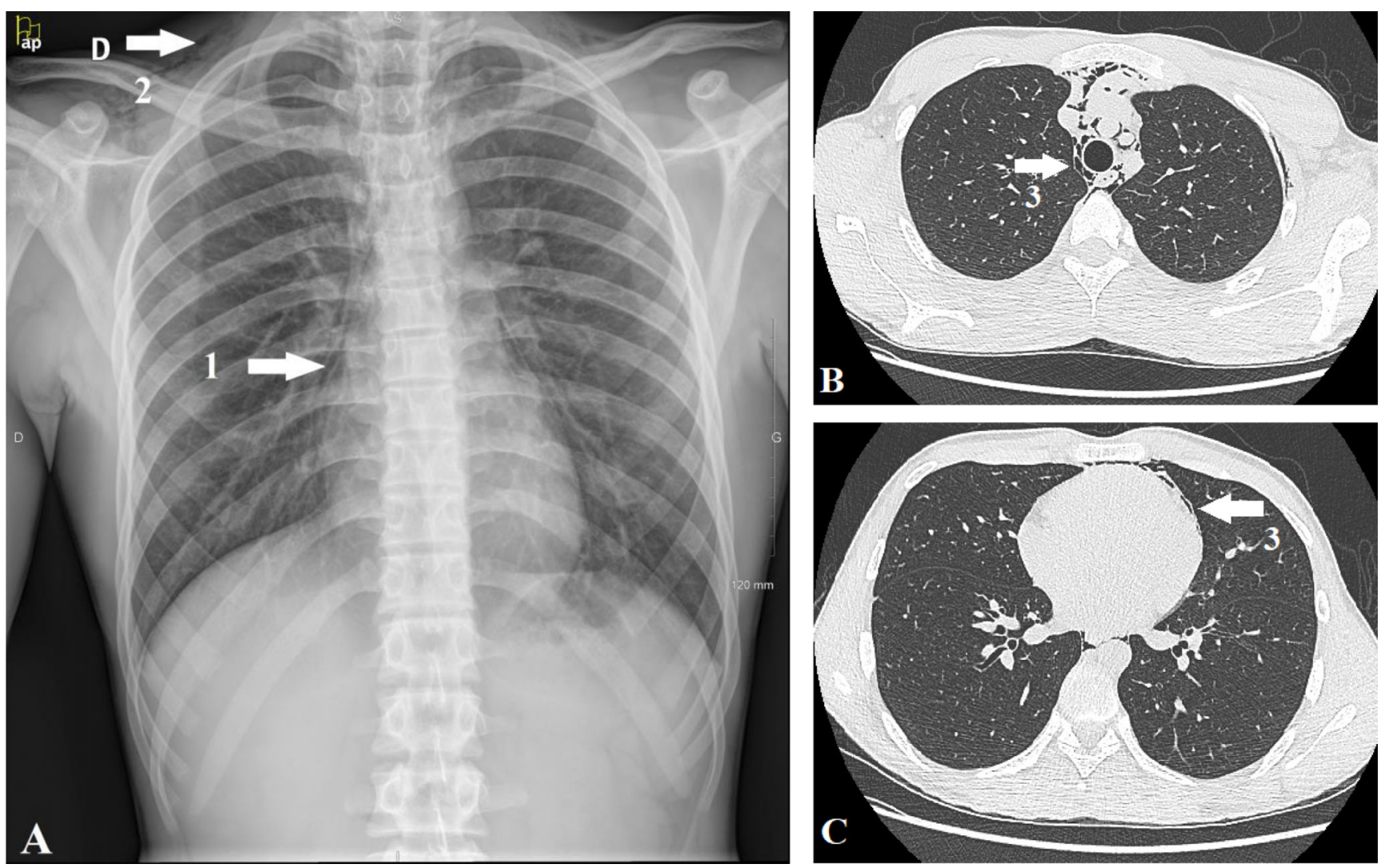

Fig. 1 A : Radiographie thoracique de face Flèche 1 : bandes d'hyperclarté des contours du cœur Flèche 2 : présence d'air sous-cutané en regard des creux axillaires droit et gauche B et C : Scanner thoracique sans injection en coupe axiale Flèche 3 : pneumomédiastin étendu de moyenne abondance, absence de pneumothorax

Le scanner confirme le diagnostic et peut permettre la mise en évidence de lésions trachéobronchiques, œsophagiennes et osseuses à l'origine du pneumomédiastin. Il permet aussi de rechercher l'association de pneumothorax. Selon l'anamnèse et le résultat du scanner, une fibroscopie bronchique à la recherche d'une lésion trachéobronchique ou un scanner avec opacification digestive à la recherche de perforation œsophagienne doit être réalisé.

La prise en charge du pneumomédiastin est avant tout le traitement étiologique s'il y a lieu. Le traitement du pneumomédiastin isolé ou spontané est seulement symptomatique.
Le pneumomédiastin traumatique est exceptionnellement compressif.

\section{Références}

1. Macklin MT, Macklin CC (1944) Malignant interstitial emphysema of the lungs and mediastinum as an important occult complication in many respiratory diseases and other conditions: an interpretation of the clinical literature in the light of laboratory experiment. Medicine 23:281

2. Mihos P, Potaris K, Gakidis I, et al (2004) Sports-related spontaneous pneumomediastinum. Ann Thorac Surg 78:983-6 